\title{
El iusnaturalismo frente a la LEY DE HUME: El CASO DE LA NEW NATURAL LAW THEORY Y SUS CRÍTICOS.
}

\author{
Felipe Widow Lira ${ }^{1}$
}

\begin{abstract}
Resumen: Según autores como Grisez y Finnis, la teoría clásica de la ley natural es inmune a la crítica contenida en el argumento de la ley de Hume, porque aquella teoría no aspira a derivar la ética de la metafísica, ni los enunciados prácticos de enunciados fácticos. La autonomía de la razón práctica, sostienen estos autores, permite una explicación de la teoría de la ley natural que no exige ningún recurso a la metafísica o a cualquier otro conocimiento teórico de la naturaleza. Esta tesis ha sido fuertemente discutida por autores vinculados al neo-tomismo que niegan la validez de la regla lógica expresada en la ley de Hume y, a la vez, afirman que la teoría tomista de la ley natural sólo se sostiene sobre sus fundamentos metafísicos. Esta discusión, sin embargo, es manifestativa de la equivocidad con que unos y otros han recibido la ley de Hume, y de la falta de análisis sobre sus supuestos y sobre el modo en que tales supuestos dialogan con la tradición clásica del iusnaturalismo.
\end{abstract}

Palabras clave: Cuestión ser-deber ser. Ley natural. Ley de Hume. New Natural Law Theory.

\section{INTRODUCCIÓN}

Durante la segunda mitad del siglo XX, la objeción contenida en la ley de Hume adquirió una progresiva importancia entre los autores iusnaturalistas de la tradición tomista, y no pocos de ellos admitieron la validez de la objeción y se dieron a la tarea de superarla mediante una nueva explicación de la teoría clásica de la ley natural ${ }^{2}$. Sólo a modo de ejemplo, se puede citar un caso que es paradigmático por el lugar que ocupa: en el artículo inaugural del

\footnotetext{
${ }^{1}$ Doctor en Filosofía por la Universidad Complutense de Madrid. Doctor en Derecho y Magister en Ciencia Jurídica por la Pontificia Universidad Católica de Chile. Licenciado en Humanidades por la Universidad Adolfo Ibánez. Profesor de Filosofía del Derecho en la Facultad de Derecho de la Pontificia Universidad Católica de Chile, Santiago, Chile. El presente trabajo se enmarca dentro del proyecto Fondecyt de Iniciación n 11171125. (D) https://orcid.org/0000-0003-2804-0920 E-mail: ffwidow@uc.cl

${ }^{2}$ Además del ejemplo de d'Entrèves, que se traerá a colación inmediatamente, y de los de Grisez, Finnis y demás autores de la New Natural Law Theory, sobre los que tratará el presente artículo, se puede mencionar otros ejemplos paradigmáticos, como los de McIntyre, Lisska o Rohnheimer.
}

http://doi.org/10.1590/0101-3173.2020.v43n3.13.p193

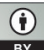

This is an open-access article distributed under the terms of the Creative Commons Attribution License. 
primer número de la revista Natural Law Forum - espacio surgido para la discusión y desarrollo de las principales tesis iusnaturalistas ${ }^{3}-$, su autor, A. P. d'Entrèves (1956, p. 29), sostiene que: " $[s]$ i queremos volver a la verdadera fuente de la distinción entre 'hecho' y 'valor', si queremos tener ante nuestros ojos un enunciado claro y franco del asunto, no podemos hacer nada mejor que recurrir a un pasaje de Hume: [...]". Y añade, tras citar el pasaje final de Treatise III, i, $1^{4}$ :

Dudo que la principal objeción al pensamiento iusnaturalista pueda ser presentada con más claridad y contundencia que en este enunciado clásico. Es la objeción a lo que se llama, en el lenguaje de los semánticos modernos, el paso del modo indicativo al modo imperativo. Una objeción, es menester admitirlo, con base en una descripción exacta de lo que los teóricos de la ley natural son, en última instancia, desde hace algún tiempo (D'ENTRÈVES, 1956, p. 30).

Y aunque - como es obvio tratándose de un autor iusnaturalista -, d'Entrèves afirmará que es posible concebir y defender una doctrina de la ley natural que salve la objeción presente en aquel texto de Hume $e^{5}$, igualmente advierte que una parte importante del pensamiento iusnaturalista ha hecho una exposición de sus tesis que no permite superar aquella objeción y que se encuentra, consecuentemente, viciada.

También John Finnis, Germain Grisez y los demás autores de la New Natural Law Theory (en lo sucesivo, NNLT) - que constituyen la más importante escuela del iusnaturalismo contemporáneo, al menos en el mundo

\footnotetext{
${ }^{3}$ En esta misma publicación aparecería, diez números más tarde, el artículo de Germain Grisez que había de iniciar el movimiento de la New Natural Law Theory. Reconvertida, luego, en The American Journal of Jurisprudence, sigue siendo, hasta el día de hoy, uno de los más importantes foros escritos sobre la teoría de la ley natural. Posiblemente el más importante del mundo anglosajón.

${ }^{4}$ El pasaje en cuestión es el siguiente: "[e]n todos los sistemas de moralidad que he encontrado hasta ahora, he notado siempre que el autor procede por algún tiempo según el modo ordinario de razonar y establece la existencia de Dios, o hace observaciones sobre las cosas humanas; mas súbitamente me sorprendo al encontrar que, en lugar de las cópulas habituales de las proposiciones, es-no es, no encuentro ninguna proposición que no esté enlazada por un debe-no debe. Este cambio es imperceptible, pero de graves consecuencias, porque este debe-no debe expresa una nueva relación, que ha de ser observada y explicada; y, al mismo tiempo, porque debe darse una razón para algo que parece completamente inconcebible: cómo esta nueva relación puede ser una deducción de otras que son enteramente diferentes de ella" (HUME, Treatise III, i, 1). Todas las traducciones presentes en este trabajo son propias, salvo que se indique expresamente lo contrario.

${ }^{5}$ Aunque lo cierto es que ni siquiera es claro que el argumento conocido como ley de Hume corresponda a la intención significativa del empirista escocés en el texto antes citado. Sobre la discusión que ha suscitado la atribución a Hume de esta ley, Vid. (WIDOW, 2015).
} 
anglosajón - admiten la validez de la objeción contenida en la ley de Hume. Un texto conjunto de Grisez, Finnis y Boyle (1987, p. 101-102) es muy claro en este punto: "[l]a teoría que defendemos también se aparta de los modelos clásicos - al menos del modo en que muchos los han entendido - porque toma plena cuenta del hecho de que el debe moral no puede derivarse del es de la verdad teorética”.

El propósito de este trabajo es describir el modo en que estos autores reciben, entienden y pretenden resolver la objeción de la ley de Hume, así como la discusión que esta recepción suscita en el medio iusnaturalista. El interés de esta discusión es, al menos, doble: en primer término, la NNLT tiene el mérito de haber reinsertado al iusnaturalismo en la discusión iusfilosófica general, y una de las razones de esta reconsideración de las tesis iusnaturalistas - por parte de autores tan distantes de ellas como pueden ser Hart, Raz o MacCormick es el hecho de la admisión e intento de superación de la objeción contenida en la ley de Hume por parte de Grisez, Finnis y compañía, dado que esta objeción había sido, a su vez, el principal motivo para considerar al iusnaturalismo como una doctrina jurídica insostenible. Esto es muy claro, por ejemplo, en Kelsen: "de un ser no podemos deducir un deber, ni de un hecho una norma; ningún deber puede ser inmanente al ser, ninguna norma a un hecho, ningún valor a la realidad empírica" (KELSEN, 1959, p. 68). Y como Kelsen entiende que es de la esencia del iusnaturalismo una tal deducción del deber a partir del ser, entonces concluye - y, con él, casi toda la filosofía del positivismo jurídico - que los intentos iusnaturalistas de fundación del derecho están inevitablemente fracasados:

La tentativa hecha por la teoría del Derecho Natural para encontrar en la naturaleza - y, consecuentemente, para deducir de ella - criterios absolutos para juzgar la elaboración del derecho positivo, es decir, normas de justicia válidas de modo absoluto, ha constituido, y debe necesariamente constituir, un fracaso, salvo que se haga un planteamiento religioso y metafísico (KELSEN, 1959, p. 148-149).

Consecuentemente, las tesis iusnaturalistas no deberían ser consideradas más que como una sofisticada expresión de la superstición en sede jurídica: "[t]oda teoría del derecho natural tiene pues un carácter religioso más o menos acentuado” (KELSEN, I960, p. 103). Es esta negativa valoración del iusnaturalismo la que cambia con la irrupción de la NNLT, al extremo de que un autor como Hart llega a exclamar, a propósito de la aparición del libro de 
John Finnis Natural Law and Natural Rights, que: "[1]a flexible interpretación de Finnis sobre el derecho natural es, en muchos aspectos, complementaria a la teoría jurídica positivista, más que un rival suyo" (HART, I983, p. 10). O Neil MacCormick, sobre el mismo libro de Finnis: "[...] nos exige abandonar nuestra versión caricaturesca de lo que es la ley natural, y nos requiere conectar argumentalmente con la fuerza real de la teoría clásica, en esta forma moderna y analíticamente sofisticada" (MACCORMICK, I98 I, p. 109). Así, pues, un primer motivo de interés para revisar la discusión sobre la recepción de la ley de Hume en la NNLT, es que esta revisión permite mirar de cerca las causas de la revaloración del iusnaturalismo que se ha descrito, y considerar si tal revaloración es correspondiente y proporcionada al tratamiento que estos autores han dado al problema de la inferencia del deber a partir del ser.

$\mathrm{Y}$ un segundo motivo de interés para la revisión que se propone este trabajo radica en su importancia para el estudio de la NNLT. Esta importancia reside en el hecho de que el modo en que es recibida la objeción contenida en la ley de Hume por Grisez, Finnis y quienes les siguen, resulta determinante de las premisas fundamentales a partir de las cuales estos autores reinterpretarán la teoría clásica de la ley natural y resolverán las cuestiones fundamentales referidas a su concepción sobre la razón práctica y su vínculo con la razón teórica o especulativa. Pues bien, como se verá en el desarrollo de este artículo, dos son las cuestiones que inquietan a los autores de la NNLT en relación con la ley de Hume: por una parte, estiman que es indispensable alejarse de afirmación - muy frecuente entre iusnaturalistas, y especialmente entre tomistas - de que la ética se halla subordinada a la metafísica. Una tal subordinación, sostienen, sería un caso flagrante de violación de la ley de Hume, ya que la ética debería consistir en una reflexión práctica sobre realidades prácticas, y subordinarla a un saber teórico como el de la metafísica la desvirtuaría, al transformarla en una conclusión práctica de premisas teóricas. Por otra parte, estos autores enfrentan la cuestión de la subordinación de la razón práctica a la especulativa y, más específicamente, del conocimiento de los primeros principios prácticos a un conocimiento especulativo de la naturaleza, lo cual también, sostienen, constituiría una inferencia ilícita de los hechos a las normas. Esta última cuestión, como se verá, es más importante que la primera puesto que, de hecho, la imposibilidad de hacer depender la ética del conocimiento metafísico estaría vinculada - en la doctrina de la NNLT - a la autonomía operativa de la razón práctica.

Estas dos cuestiones determinan los objetivos y el orden de este trabajo: en primer lugar, se expondrá la postura de la NNLT respecto de la relación 
entre ética y metafísica, así como las críticas que tal postura ha suscitado en el ambiente iusnaturalista. En segundo lugar, se revisarán los argumentos en virtud de los cuales estos autores sostienen la autonomía de la razón práctica y la correspondiente inderivabilidad de los primeros principios prácticos, desde los cuales una teoría de la ley natural podría superar la objeción contenida en la ley de Hume, e igualmente las críticas que estos argumentos han recibido de otros autores iusnaturalistas. La conclusión de este trabajo aspira a ser una constatación que emerge de la revisión de estas dos discusiones: que la asunción de la validez de la ley de Hume y de su fuerza para objetar la teoría clásica de la ley natural es, tanto en los autores de la NNLT como en muchos de sus contradictores, superficial y acrítica: Grisez y Finnis adoptan las tesis de algunos analíticos de segunda generación, como Hare y Nowell-Smith, sin detenerse a considerar los supuestos que se hallan en las bases de esas tesis, ni las discusiones que las mismas han suscitado en el seno de la propia filosofía analítica, y lo mismo sucede, en mayor o menor medida, con sus críticos tomistas.

\title{
1 ¿Es LíCITO DERIVAR LA ÉTICA DE LA METAFísica?
}

Cuando, en 1965, Germain Grisez se propone presentar una nueva interpretación de Prima Secundae 94, 2, de la Suma Teológica de Tomás de Aquino, comienza haciendo la siguiente advertencia:

\begin{abstract}
Muchos defensores y críticos de la teoría de Tomas de Aquino sobre la ley natural la han entendido, aproximadamente, como sigue: el primer principio de la razón práctica es un mandato: 'Haz el bien y evita el mal'. El hombre descubre este imperativo en su conciencia; él es como una inscripción escrita allí por la mano de Dios. Habiendo alcanzado el conocimiento de este mandato básico, el hombre consulta su naturaleza para ver qué es bueno y qué es malo. Examina una acción en comparación con su esencia para ver si la acción se ajusta o no a la naturaleza humana. Si la acción se ajusta, aparece como buena; si no se ajusta, aparece como mala (GRISEZ, 1965, p. 168).
\end{abstract}

Y luego añade: "[y]o me propongo mostrar cuán lejos está esta interpretación de la posición real del Aquinate” (GRISEZ, 1965, p. 168). Y, aunque todo el artículo discurre, dialécticamente, entre la demostración del carácter erróneo de aquella interpretación y la nueva interpretación que propone Grisez, lo interesante, en este punto, es advertir que aquella interpretación errónea 
no es sostenible, precisamente, porque incurre en el defecto que denuncia la ley de Hume, al pretender derivar los principios morales desde juicios metafísicos sobre el conocimiento de la naturaleza humana: "para obtener principios morales desde la metafísica, no debemos transitar desde el 'es' de la naturaleza hasta el 'debe' de la naturaleza. Esta deducción [...] no es de ayuda en la explicación del origen de los juicios morales" (GRISEZ, 1965, p. 194).

Con lo cual advierte, a la vez, sobre dos posibles errores en la concepción de lo que la ley natural es, y del modo en que se presenta al conocimiento y dirige la acción moral. Uno de esos errores - el más explícito en el texto citado - sería la subordinación de la ética a la metafísica. Si el fin de la ética es señalar los criterios necesarios para determinar la bondad o malicia de nuestros actos libres, entonces la metafísica no podría ayudarle en ello, porque semejante finalidad práctica no cabe, de ningún modo, en el espectro estrictamente reflexivo o especulativo de los juicios metafísicos: "[l]a invocación a la metafísica de la causalidad y la providencia divinas no ayuda en este punto, porque tal metafísica consiste, exclusivamente, en verdades teóricas, desde las cuales la razón no puede derivar consecuencias prácticas" (GRISEZ, 1965, p. 198).

Esta postura contraria a la subordinación de la ética a la metafísica no es, entre los autores iusnaturalistas, original de Grisez. El propio d'Entrèves explica, al señalar una de las formas más difundidas de la que él considera una errónea exposición de la doctrina del Derecho Natural, que tal error consistiría en buscar el fundamento del Derecho en la 'ontología':

Una manera de concebir el Derecho Natural es aquella que se da a sí misma el nombre de 'ontología' y se presenta como 'ciencia del ser' del derecho, del cual afirma que es posible obtener pleno conocimiento mediante la metafísica del ser. Ser y deber ser, según esta concepción, no están separados - como lo pretende Hume - por un abismo insalvable. Por el contrario, es del conocimiento del ser que deriva toda noción de deber ser [...] La objeción más grave para su aceptación reside, en mi parecer, precisamente en la premisa metafísica sobre la que se apoya y de la cual obtiene su lógica impecable (D’ENTREVES, 1959, p. 154-155) ${ }^{6}$.

Así, pues, al parecer, la ley de Hume pondría en un grave aprieto a esas extendidísimas escuelas iusnaturalistas que situaban el fundamento de la moralidad en el orden metafísico y que, de este modo, entendían que una lectura filosófica de la ley natural equivalía a una reflexión metafísica

${ }^{6}$ Expresiones muy semejantes se pueden encontrar en otras obras del mismo autor, como en Natural Law. An Introduction to Legal Philosophy. (D'ENTREVES, 1951). 
sobre la misma. Un autor que luego entraría en discusión con la NNLT, Lawrence Dewan, sintetiza nítidamente esta posición: "[l]o que significamos por 'la doctrina tomista de la ley natural' es la reflexión metafísica sobre la naturaleza de nuestro conocimiento de los primeros principios prácticos, y la descripción metafísica de nuestro originario conocimiento natural de esos principios" (DEWAN, 1990, p. 286) ${ }^{7}$. Esto sería, según una posición como la de d'Entrèves, exactamente aquello en lo que no puede consistir la ética - ni la doctrina de la ley natural - si no quiere quedar presa de la objeción que ofrece la ley de Hume. Quedarían singularmente señaladas las escuelas llamadas neotomistas, que tanta difusión habían alcanzado en los finales del siglo XIX y la primera mitad del XX. Y en tal visión crítica del tomismo imperante se situaban, también, los autores de la escuela de la NNLT.

Esta actitud aparentemente 'anti-metafísica' habría de generar fuertes y apasionadas reacciones entre otros defensores tomistas de la ley natural - pertenecientes a aquellas escuelas neo-tomistas -, como se advierte en las discusiones que, en el mundo anglosajón, siguieron a la publicación de Natural Law and Natural Rights (Vid. SCHULTZ, 1985) y en las que intervinieron activamente autores como Ralph McInerny (Vid. 1980, 2000), Henry Veatch (Vid. 1981), Russell Hittinger (Vid. 1987) o el propio Lawrence Dewan (Vid. 1990). Este último expone muy claramente la lectura que estos autores hacían de los intentos de Grisez y Finnis:

Finnis quiere presentar un argumento genuinamente ético, que se diferencie de una reflexión metafísica sobre la acción humana y las ciencias humanas, y quiere hacerlo así mediante un uso considerable de las argumentaciones éticas presentes en los escritos de Santo Tomás. Esto le obliga a decir que toma lo que es esencial a la argumentación ética de Santo Tomás, y deja lo que es 'un apéndice especulativo ańadido a esta argumentación por medio de la reflexión metafísica'. Estos son juicios arriesgados [...] (DEWAN, 1990, p. 285).

Como dice Beuchot, la discusión engendrada por los escritos de Grisez y Finnis traza una línea divisoria entre dos tipos de autores iusnaturalistas que creen reconocer en Santo Tomás a la autoridad que les da soporte y base doctrinal: "algunos autores extraen la ley natural de la naturaleza humana, y otros pretenden sacarla de los primeros principios de la razón práctica” (Beuchot, 1992, p. 19), y tanto los unos como los otros estiman falsa la tesis contraria, a la vez que infiel a la auténtica filosofía del Aquinate. A los que

\footnotetext{
${ }^{7}$ Lisska se pronuncia en términos muy semejantes. Vid. (LISSKA, 1996, p. 152 y ss).
} 
procuran sacar la ley natural de los primeros principios prácticos (NNLT), "les parece que lo primero [sacarla de la naturaleza] es cometer la falacia naturalista” (BEUCHOT, 1992, p. 19), los otros, por el contrario, entienden que "sólo el metafísico puede decir si la ley natural deriva el ser del deber ser y si eso es lícito" (BEUCHOT, 1992, p. 20). De aquí que reaccionen duramente contra el hecho de ser acusados de cometer aquella falacia por unos autores que se cierran a priori a aquella perspectiva metafísica ${ }^{8}$. La más extrema de estas reacciones ha sido, quizá, la de Henry Veatch, quien, en un artículo de 1981, llega a plantear preguntas como la siguiente:

¿Puede uno extender, legítimamente, la imposibilidad de inferir la ética desde la metafísica a la conclusión, de algún modo sugerida por Finnis, de que la ética no puede tener base en la metafísica de ningún modo, o de que las consideraciones metafísicas han de ser consideradas irrelevantes incluso cuando hacen inteligibles los principios éticos? (VEATCH, 1981, p. 254).

O, al final del mismo artículo: “¿[e]s necesario decir más sobre el muro de separación que tanto Grisez como Finnis sostienen que existe entre la razón práctica y la teórica, entre la ética y la metafísica, entre la naturaleza y la moral, entre 'es' y 'debe'?" (VEATCH, 1981, p. 265). Preguntas, éstas, que motivan una no menos calurosa respuesta de los autores interpelados y que permiten una precisión del sentido en que los fundadores de la NNLT afirman aquella independencia de la ética respecto de la metafísica. No se trata - dirán Grisez y Finnis - de negar el valor de la metafísica iluminando la ética, ni la posibilidad de reflexionar teóricamente - metafísicamente - sobre cuestiones que son prácticas - éticas -. De lo que se trataría, según la postura que ellos defienden, es de reconocer que los fundamentos desde los cuales es posible todo el despliegue argumental de la ética no son metafísicos, porque no tienen que ver con los hechos de la naturaleza sino con los principios de la razón práctica. Por ello, sin negar que la reflexión metafísica podría otorgar una visión de conjunto sobre la naturaleza humana, en la que se integra el orden práctico o moral, sería menester afirmar que es posible una visión parcial - que, en este caso, correspondería a la ética - que otorgaría explicaciones válidas y verdaderas sobre la dimensión moral de la vida de los hombres, y que esta visión parcial sería accesible sin aquella visión de conjunto:

\footnotetext{
${ }^{8}$ Una síntesis de los elementos centrales de cada una de estas dos tesis se puede encontrar en Antonella Corradini (2003).
} 
Estoy a favor de la metafísica como parte de (y, en cierto sentido, la parte fundamental de) la gran búsqueda por la clarificación y la explicación. Dediqué más de un capítulo de mi libro sobre la ley natural a esta sola clase de explicación. La llamé, con franqueza, 'no práctica, sino teórica o metafísica', y reclamé que las respuestas a las cuestiones teóricas o metafísicas allí planteadas fuesen reconocidas como necesarias si en ellas se encontraba cualquier respuesta completamente satisfactoria a las profundas preguntas prácticas sobre el tema de los bienes humanos. También reclamé - y esto es lo que Veatch parece objetar - que, así como 'puede darse una buena explicación del movimiento molecular' sin la explicación de todo el universo o de la dependencia del universo y del movimiento molecular respecto de la causa incausada, 'así, también, (...) la ley natural puede ser entendida, aceptada, aplicada y reflexivamente analizada' sin explorar las cuestiones metafísicas a las que me había referido (FINNIS, 1981, p. 275276).

De este modo, el primero de los errores que denunciaba Grisez - en el párrafo más arriba citado - no consistiría en valorar las explicaciones que, sobre la naturaleza, eventualmente aporte la metafísica, ni en aprovechar las luces que el análisis metafísico pueda dejar caer sobre las cuestiones prácticas. El error consistiría en reducir los principios de la ética a fórmulas metafísicas, haciendo así imposible una explicación del orden moral desde el solo orden práctico. Se podría admitir que la eventual demostración de la existencia de Dios, por ejemplo, y su consideración como causa ordenadora y final de todo el universo, aportará muchas clarificaciones que habrán de ser integradas en los dominios de la ética; no obstante, lo que resulta inadmisible es la afirmación de que la ética sea dependiente de tales enunciados metafísicos. En otras palabras, y conectando esta cuestión con la ley de Hume, Grisez, Finnis y, en general, los autores de la NNLT, manifestarán su acuerdo con la afirmación de unos vínculos muy estrechos entre la ética y la metafísica, pero negarán rotundamente que la primera se derive de la segunda. Esta sería la clave lógica del problema y, si se intentara tal derivación, entonces se invalidarían los argumentos que procuran sostener la doctrina de la ley natural, porque se los haría culpables de una inferencia ilícita del orden teórico al orden práctico.

\section{2 ¿ES LÍCITO INFERIR ENUNCIADOS PRÁCTICOS A PARTIR DE JUICIOS ESPECULATIVOS?}

Pero no sería éste el error más grave, a juicio de Grisez, ni al que le concede mayor atención: detrás de la reducción de la ética a la metafísica se 
escondería una deformidad mayor: la reducción del conocimiento práctico al conocimiento teórico, la confusión de la razón práctica con la especulativa, el reemplazo, en definitiva, de la razón en cuanto directiva de la acción, por un conocimiento reflexivo de la naturaleza o esencia humana:

Si alguien supone que los principios de la ley natural se forman mediante el examen de clases de acciones en comparación con la naturaleza humana y advirtiendo su mutua conformidad o disconformidad, entonces se debe responder a la objeción de que es imposible derivar juicios normativos desde especulaciones metafísicas (GrisEz, 1965, p. 196).

Y no sería posible responder satisfactoriamente a esta objeción, porque ella plantea una regla lógica inquebrantable: aquella que indica que de enunciados fácticos no se pueden concluir juicios morales o, como afirma rotundamente Finnis (2011b, p. 50): que "[l] a inferencia del 'es' al 'debe' es evidentemente falaz”. O, como advierten Grisez, Finnis y Boyle (1987, p. 102): "[...] de un conjunto de premisas teóricas no se puede derivar lógicamente ninguna verdad práctica, porque un razonamiento válido no introduce lo que no está en las premisas".

Esto, en el ámbito de la doctrina iusnaturalista, se traduce en que la fundación práctica del deber no podría hallarse en la referencia del mismo a la naturaleza humana, o al orden que Dios ha puesto en las criaturas y, específicamente, en el hombre. En otras palabras: "de la conformidad de las acciones con la naturaleza humana, o con un decreto de la voluntad divina, no se puede derivar la sentencia prescriptiva: 'esto debe hacerse'" (GRISEZ, 1965, p. 194). Esta traducción de aquella regla lógica a un lenguaje iusnaturalista es interesante porque, a la vez, no se ha perdido de vista la identidad de la regla en cuestión con su formulación analítica, de manera que puede comenzar a advertirse que los juicios que expresan el conocimiento de la naturaleza humana, o de los decretos de la voluntad divina, se hallan en el ámbito de lo que analíticos como Hare o Nowell-Smith habrían llamado 'enunciados fácticos'.

Que al plantear tal regla lógica - como una objeción insalvable para ciertas exposiciones de la doctrina de la ley natural - tenían en mente la misma regla lógica que aquellos autores analíticos habían formulado - y atribuido a Hume -, es algo expreso en muchos pasajes, singularmente en uno de Grisez anteriormente citado: dice, el franco-americano, al tratar del

\footnotetext{
9 Sobre el sentido de la ley de Hume en la segunda generación de filósofos morales analíticos (Hare, Nowell-Smith, Ayer, etc.), Vid. (WIDOW, 2016-2017).
} 
defecto de aquellas erróneas interpretaciones de I-II, q. 94, a. 2 in c.: "[e]ste es, precisamente, el punto que Hume vio cuando negó la posibilidad de derivar el deber a partir del ser" (GRISEZ, 1965, p. 194). Y que no hay equívocos en el modo de comprender esta regla - respecto de la comprensión que de ella habían hecho autores como Hare o Nowell-Smith -, es algo que se manifiesta con una patencia aún mayor en el lenguaje que Grisez y Finnis (2011c, p. 78) emplean para explicarla: dice el australiano: “[...] estoy completamente satisfecho de enfatizar la diferencia entre juicios 'fácticos' y juicios 'valorativos', y de afirmar que los últimos no se derivan por inferencia de los primeros, sino que son sui generis", de modo que la dificultad para poner el fundamento del deber en el conocimiento de la naturaleza humana, o en el reconocimiento de la conformidad de nuestras acciones con aquella naturaleza, radicaría en el hecho de que aquél conocimiento se expresa en un tipo de enunciados o juicios radicalmente diversos de aquellos que expresan el deber moral. La imposibilidad de la inferencia de unos a otros, en consecuencia, remite primariamente al hecho de que las diversas formas enunciativas que los diferencian supone en ellos la presencia de elementos no comunicantes. Es, en el lenguaje de Hare, el néustico del enunciado prescriptivo - esto es, la condición de estos juicios de sugerir o dirigir una acción -, el que no se hallaría por ninguna parte en el enunciado fáctico, como se advierte nítidamente en el siguiente pasaje de Finnis (2011a, p. 242):

Hume trató como una novedad admirable su idea de que 'debe' no puede ser derivado de 'es' (que ignora u omite en su propio trabajo positivo en ética y metaética). Pero hubo filósofos anteriores que habían entendido mejor que el deber moral, la dirección de la razón en la deliberación para la elección y la acción, no se puede derivar a partir de (o reducirse a) el 'es' de la naturaleza, o a la lógica de la no-contradicción.

Sería, así, 'la dirección de la razón en la deliberación para la elección y la acción' la que se hace presente en el 'enunciado valorativo', y la que no cabría en el 'enunciado fáctico' sobre la naturaleza. Lo que está en juego, para estos autores - como lo estaba para los analíticos que formularon la regla -, es la autonomía de la razón práctica que, si ha de ser auténticamente práctica, debe tener principios propios desde los cuales desplegar toda su operación racional, sin depender en nada - al menos no intrínsecamente - del conocimiento teórico que se pueda alcanzar mediante la dimensión especulativa del entendimiento: "[...] los últimos principios de la moralidad no pueden ser verdades teoréticas de la metafísica o de la antropología filosófica” (GRISEZ et al., 1987, p. 102). 
Esto lo afirma muy claramente Grisez al adherirse a cierto aspecto de la crítica que dirigiese Kai Nielsen (1959) contra el iusnaturalismo tomista, en el supuesto de que en tal corriente de pensamiento se confundirían las categorías del orden práctico - entre las que se encuentran los valores - con aquellas del orden teórico - que corresponden a los hechos - (aunque el franco-americano advierte que lo que Nielsen critica no son tesis que defienda el auténtico Santo Tomás, sino deformaciones posteriores de su doctrina de la ley natural):

En un interesante pasaje de un artículo en el que ataca lo que, erróneamente, considera como la teoría de la ley natural de Santo Tomás, Kai Nielsen discute este punto con cierta amplitud. Comienza argumentando que los enunciados normativos no se pueden derivar de enunciados de hecho, ni siquiera de un conjunto de enunciados de hecho que comprendan una verdadera teoría metafísica de la realidad. Señala que desde 'Dios quiere x', no se puede derivar "x es obligatorio" sin asumir la premisa no fáctica "lo que Dios quiere es obligatorio". Continúa criticando lo que toma como una confusión, en el tomismo, entre hecho y valor, una combinación de categorías dispares, que Nielsen considera ininteligible. Pero más allá, y por encima de esta objeción, insiste en que el discurso normativo, en la medida en que es práctico, simplemente no puede derivarse de meras consideraciones de hechos (GRISEZ, 1965, p. 194).

Y el único modo de evitar esta confusión de categorías - y rescatar, así, la autonomía operativa de la razón práctica - sería la afirmación de que todo el discurso normativo o valorativo se constituye de juicios que, como indicase Finnis, son 'sui generis', es decir, que pertenecen a un género diverso de aquellos referidos a 'hechos', y esto sólo sería posible si los principios de tales juicios son conocidos absolutamente por la razón práctica y de ningún modo dependen de alguna actividad especulativa: "[1] os principios sobre lo que es moralmente correcto o incorrecto se derivan de los primeros principios premorales de razonabilidad práctica, y no de algunos hechos, sean metafísicos o de otro tipo" (FINNIS, 2000, p. 67).

Esta postura también había de generar incomprensiones en el medio iusnaturalista. Y también estas incomprensiones facilitarían importantes precisiones en la doctrina de la NNLT sobre el tópico. Ya los textos citados de Henry Veatch permiten advertir por qué camino van las objeciones: en una primera lectura de la doctrina de Grisez y Finnis, pareciera que el orden práctico es completamente indiferente a la realidad del mundo tal como ésta se presenta a la razón especulativa, y que se puede obrar moralmente sin consideración alguna de los hechos que rodean y condicionan nuestras 
acciones. Esta acusación la deja caer, sin demasiados matices, Ralph McInerny (1980, p. 11), al sostener que la visión de Grisez y Finnis no es tomista: “[u] na visión de la razón práctica que considera irrelevante el conocimiento del mundo es, claramente, una visión diferente de aquella que encontramos en Aristóteles y Santo Tomás", irrelevancia que, sostiene, se sigue de una "excesiva distinción entre hecho y valor" (MCINERNY, 1980, p. 7), lo cual "sugiere que hay algo ilícito en el paso de una proposición como: 'Los cereales son buenos para ti' a 'Debes comer cereales'” (MCINERNY, 1980, p. 11). Pero Grisez y Finnis (1981, p. 22-23) rechazan firmemente esta acusación:

\begin{abstract}
No piensa Finnis que 'el conocimiento del mundo sea irrelevante para la razón práctica', ni siquiera para la comprensión, por la propia razón práctica, de sus principios básicos. Explicando lo que él entiende por 'los valores no pueden derivarse de los hechos', dice: [cita de Natural Law and Natural Rights] '...mi argumento es que, aunque el conocimiento de ciertas posibilidades 'de hecho' es una condición necesaria para el juicio razonable de que la verdad es un valor, aun así, ese mismo juicio no se deriva de otro juicio cualquiera'.
\end{abstract}

En realidad, en esta cuestión los fundadores de la NNLT piensan de un modo semejante a Hare o Nowell-Smith - y a Hume, tal como lo leen estos autores analíticos - respecto de la posibilidad de integrar enunciados de hecho en razonamientos prácticos. Como es bien sabido, Hume criticaba la tesis de Wollaston - que sostenía que es posible reducir todo el mal moral a la mentira porque en toda acción inmoral hay una cierta falsedad - acusándolo de que no justificaba que la mentira fuese un mal moral (HUME, Treatise III, i, 1, note 66), de manera que intentaría deducir deberes morales ('No se debe hacer $X$ ) desde constataciones puramente fácticas (' $X$ es mentira'). A partir de esto, aquellos autores analíticos (NOWELL-SMITH, 1977, p. 53-56; HARE, 1975, p. 46-47) sostenían que la doctrina de Hume - que hacían suya - consistía en que, desde el enunciado de hecho ' $X$ es mentira', sólo podría concluirse que 'No se debe hacer $X$ ' si el razonamiento estaba fundado en un principio que dijese 'No se debe mentir', y que tal principio debía ser, a su vez, justificado mediante algún razonamiento anterior, porque de otro modo persiste el problema de la fundamentación última del orden práctico, que es el problema que Hume habría advertido en Wollaston: "[c]oncederé, si así se quiere, que toda inmoralidad consiste en esta supuesta falsedad de la acción, pero exijo, como única condición, que se me presente una razón plausible de por qué semejante falsedad es inmoral" (HUME, Treatise, III, i, 1, note 66). 
Que Grisez y Finnis conciben del mismo modo la posibilidad de integrar enunciados de hecho en razonamientos prácticos, es algo que queda patente en la respuesta que dan al ejemplo propuesto por McInerny, referente a la posibilidad de deducir 'Debes comer cereales' desde el enunciado fáctico 'Los cereales son buenos para ti' ${ }^{10}$. De este modo responden los interpelados:

Si McInerny desea justificar una conclusión como 'Joe debe hacer una dieta', es mejor no argumentar con premisas tales como 'Joe pesa 225 libras' y 'No es saludable estar obeso'. Se debe asumir una premisa práctica más básica: 'La salud es un bien que debe ser perseguido y protegido', que es una especificación del mismísimo primer principio de la razón práctica (FINNIS; GRISEZ, 1981, p. 24).

La identidad entre el razonamiento propuesto por Grisez y Finnis y aquel defendido por Hare y Nowell-Smith es total. Y también hay tal identidad en la consideración del problema esencial para la ética que esconde ese razonamiento: si se aspira a no cometer nunca el salto ilícito desde enunciados de hecho a proposiciones prácticas, entonces es necesario encontrar una premisa absolutamente primera que sea práctica. Sólo así estará asegurada la no dependencia de la razón práctica respecto de la especulativa. En la ética analítica éste ha sido, precisamente, el punto de dispersión entre intuicionistas, emotivistas y prescriptivistas. La doctrina iusnaturalista de la NNLT cree encontrar la solución de esta cuestión en la referencia a unos bienes humanos básicos auto-evidentes, que se presentan en el razonamiento práctico - como lo advierte el texto citado - como objeto de principios y preceptos que son especificaciones de los primeros principios y, singularmente, del primer principio de la razón práctica, sobre el que se fundan todos los demás.

La clave de bóveda de esta solución será, consecuentemente, el modo en que se entienda este primer principio de la razón práctica. Y, en orden a salvaguardar la autonomía de la razón práctica, es este principio el que debe, sobre todo, conservar su independencia respecto de los 'enunciados fácticos' que aprehende la razón especulativa: “El bien ha de ser hecho y perseguido, y el mal ha de ser evitado', junto con los otros principios auto-evidentes de la ley natural, no se derivan de ningún enunciado de hecho" (GRISEZ, 1965, p. 195).

${ }^{10}$ En realidad, este sería un caso de lo que Hare llamaría un enunciado encubiertamente valorativo, porque en la nocion de 'bueno para tí' se esconde, ya, un cierto lenguaje moral. (Hare, 1975, p. 9495). 
Según Grisez y Finnis, una lectura atenta de la teoría ética de Aristóteles, así como de la doctrina iusnaturalista de Santo Tomás, mostraría que esta independencia de la razón práctica en el conocimiento de sus primeros principios y, consecuentemente, en toda su operación posterior, se halla en el núcleo de sus construcciones filosófico morales, de manera que las mismas no se verían afectadas por la objeción contra los naturalismos planteada en la ley de Hume. En otras palabras, la recta interpretación de aquellos autores clásicos indicaría que ellos no han cometido el error de omitir la diferencia lógica entre enunciados fácticos y valorativos ni, en consecuencia, el de inferir los unos a partir de los otros: "Aristóteles y Tomás de Aquino admitirían sin dificultad que el deber no puede deducirse del ser" (FINNIS, 2000, p. 80).

Por ello - dirían los fundadores de la NNLT y los autores que se han asociado a sus tesis centrales -, una doctrina iusnaturalista que aspire a sortear las dificultades que podría suponer esta objeción no debe procurar demostrar que la ley de Hume es una regla viciada o mal formulada, ni pretender que la solución del problema pasa por tender un puente sobre el abismo que media entre el ser y el deber. En otras palabras, sería necesario reconocer que la doctrina de la ley natural no es destinataria de la objeción contenida en la ley de Hume, y huir de dilemas como el que propone Veatch (1981, p. 253): "[...] se deben generar las condiciones que permitan proceder de los hechos a las normas, o dejar de intentar abrazar la filosofía de la ley natural”. Bastaría, simplemente, con comprender adecuadamente la naturaleza de la razón práctica y, atendida su autonomía operativa, poner en ella el fundamento de la moralidad humana, y no en un conjunto de hechos, metafísicos o de cualquier otra especie. Es así que, frente a la pregunta de Julius Stone: “¿[h]an mostrado los iusnaturalistas que ellos pueden derivar normas éticas a partir de hechos?”, Finnis responde con gran rotundidad: "no lo han hecho, no necesitan hacerlo, ni los exponentes clásicos de la teoría soñaron con intentar alguna derivación de este tipo" (FINNIS, 2000, p. 66).

\section{Conclusiones}

La discusión reseñada muestra de un modo muy claro los condicionamientos que el pensamiento moderno impone a las reformulaciones contemporáneas de la teoría clásica de la ley natural. En efecto, la disputa entre los defensores y los críticos de la NNLT pone en evidencia que hoy no es posible proponer teóricamente la tesis de una ley natural sin hacerse cargo 
de las dificultades que entrañan objeciones como la que se contiene en el argumento de la ley de Hume. No obstante, esta discusión muestra, también, que hay un gran peligro de caer en contradicciones internas cuando se asume un argumento u objeción de una tradición filosófica ajena a la propia, sin detenerse a considerar todos los supuestos implícitos de aquel argumento u objeción. Esto, a nuestro juicio, sucede con la reformulación de la teoría de la ley natural que intentan Grisez y Finnis, y también - aunque de otro modo con algunos de sus críticos.

En efecto, cuando los autores de la NNLT afirman la imposibilidad de derivar la ética de la metafísica, o cualquier juicio práctico de un juicio especulativo, lo hacen como si esta distinción, en el contexto de la teoría clásica de la ley natural, fuera equivalente a la distinción que algunos analíticos habían establecido entre enunciados prácticos y enunciados fácticos. E incluso cuando alguno de los críticos de la NNLT afirma lo contrario por ej. Henry Veatch (1981, p. 253) al decir que "se deben generar las condiciones que permitan proceder de los hechos a las normas" - y vincula esta afirmación con la posibilidad de derivar la ética de la metafísica, también él razona como si los "hechos" de aquellos analíticos fueran equivalentes a la "naturaleza" de la teoría clásica de la ley natural, o los "enunciados fácticos" a las "proposiciones metafísicas sobre la naturaleza" de aquella misma teoría. Ni unos ni otros realizan un examen suficientemente detenido de los problemas y equivocidades que se generan al intentar un diálogo entre tradiciones filosóficas tan distintas. Sólo a modo ejemplar, se pueden enumerar algunas de las cuestiones que exigirían un análisis más detallado, antes de proponer una solución iusnaturalista al problema de la inferencia del deber a partir del ser: ¿es la naturaleza de la metafísica aristotélico-tomista expresable en términos de hechos, tal como estos son entendidos por autores como Hare o NowellSmith? ¿se puede afirmar que en las tesis de estos últimos hay antecedentes metafísicos o gnoseológicos nominalistas y/o empiristas? De ser así, ¿̨cuál sería la mediación necesaria para traducir la regla lógica de la ley de Hume, tal como la formulan estos analíticos, al contexto metafísico y gnoseológico de la teoría clásica de la ley natural? Puestos a asumir los desarrollos lógicos de la teoría moral analítica, ¿no sería conveniente detenerse en las discusiones que la ley de Hume ha generado en la propia escuela analítica? Las propuestas teóricas de autores como Searle o Gewirth, que ponen en cuestión el argumento de la ley de Hume, al menos en el sentido defendido por Hare y Nowell-Smith ¿̨no ofrecen ninguna consideración relevante para el diálogo entre la teoría clásica de la ley natural y la filosofía analítica? Por supuesto, no es que estas cuestiones pasen 
completamente inadvertidas a los autores trabajados en este artículo. Pero sí que es cierto que no han recibido la dedicación y el tratamiento proporcional a la importancia que la asunción de la ley de Hume tiene en la NNLT, ni en la crítica que tal asunción suscita en otros autores iusnaturalistas.

A nuestro juicio, cuando Grisez y Finnis procuran la reformulación de la teoría de la ley natural en unos términos que evidencien su inmunidad frente a una objeción como la ley de Hume muestran su implícita (y, a veces, explícita) dependencia (al menos metodológica, aunque por momentos parece también gnoseológica y metafísica) de unas tesis analíticas que no tiene plenamente integradas en su sistema (ni que, posiblemente, podrían integrar sin dejar de autodenominarse tomistas o iusnaturalistas clásicos). Y cuando sus críticos sostienen la invalidez de la objeción, en razón de la afirmación sin matices de la licitud de la derivación de los hechos a las normas, también muestran una implícita (y, a veces, explícita) dependencia de una comprensión particular de la teoría clásica (ambigua y compleja, en muchos aspectos, y que hunde sus raíces en la lectura que autores como Suárez y Vázquez hicieron de Tomás de Aquino), en la que se han introducido elementos igualmente contradictorios con los fundamentos de esa teoría en sus autores clásicos.

WIDOW, $\mathrm{F}$. Iusnaturalism in front of hume's law: the case of the new natural law theory and its critics. Trans/form/ação, Marília, v. 43, n. 3, p. 193-212, Jul./Set., 2020.

\begin{abstract}
According to authors such as Grisez and Finnis, the classical theory of natural law is immune to the criticism contained in Hume's law argument, because that theory does not pretend to derive ethics from metaphysics, nor practical statements from factual statements. These authors argue that the autonomy of practical reason allows an explanation of the theory of natural law, which does not require any recourse to metaphysics or any other theoretical knowledge of nature. This thesis has been strongly contested by authors linked to neo-Thomism, who deny the validity of the logical rule expressed in Hume's law and, at the same time, affirm that the Thomistic theory of natural law can only be sustained on its metaphysical foundations. This discussion, however, is indicative of the ambiguity with which they have received Hume's law, and of the lack of analysis on the assumptions of such law and on the way in which such assumptions dialogue with the classical tradition of natural law.
\end{abstract}

Keywords: Is-ought question. Natural Law. Hume's law. New Natural Law Theory. 


\section{Bibliografía}

BEUCHOT, M. Naturaleza humana y ley natural como fundamentos de los derechos humanos. Ethos, 19-20, 1992.

CORRADINI, A. On the Normativity of Human Nature: Some Epistemological Remarks. Journal of Medicine and Philosophy, vol. 28, n. 2, 2003.

D’ENTRÈVES, A. P. Le Droit Naturel. In: AA. VV. Le Droit Naturel. Paris: Presses Universitaires de France, 1959.

D’ENTRÈVES, A. P. The Case for Natural Law Re-Examined. Natural Law Forum, 1, 1956.

DEWAN, L. St. Thomas, Our Natural Lights and the Moral Order. Angelicum, 67, 1990.

FINNIS, J.; GRISEZ, G. The Basic Principles of Natural Law: a Reply to Ralph McInerny. The American Journal of Jurisprudence, 26, 1981.

FINNIS, J. Commensuration and Public Reason. In: John FinNIs. Reason in Action. Collected Essays: Volume I. Oxford: Oxford University Press, 2011a.

FINNIS, J. Discourse, Truth, and Friendship. In: John FInNIs. Reason in Action. Collected Essays: Volume I. Oxford: Oxford University Press, $2011 \mathrm{~b}$.

FINNIS, J. Scepticism's Self-Refutation. In: John FinnIs. Reason in Action. Collected Essays: Volume I. Oxford : Oxford University Press, 2011c.

FINNIS, J. Ley natural y derechos naturales. Traducción y estudio preliminar de Orrego, C. Abeledo-Perrot, Buenos Aires, 2000.

FINNIS, J. Natural Law and the 'Is'-'Ought' Question: an Invitation to Professor Veatch. Catholic Lawyer, n. 26, 1981.

GRISEZ, G., BOYLE, J., FINNIS, J. Practical Principles, Moral Truth and Ultimate Ends. The American Journal of Jurisprudence, 32, 1987.

GRISEZ, G. The First Principle of Practical Reason: A Commentary on the Summa Theologiae, I-II, Question 94, Article 2. Natural Law Forum, 10, 1965.

HARE, R. M. El lenguaje de la moral. Traducción de Carrió, G. y Rabossi. México: E. UNAM, 1975.

HART, H. L. A. Essays in Jurisprudence and Philosophy. Clarendon Press, Oxford, 1983.

HITTINGER, R. A Critique of the New Natural Law Theory. Notre Dame - Indiana: University of Notre Dame Press, 1987.

HUME, David. A Treatise of Human Nature. Edición de Selby-Bigge, L. A. Oxford: Clarendon Press, 1896. 
KELSEN, H. Teoría pura del derecho. Introducción a la ciencia del derecho.

Traducción de NILVE, M. Buenos Aires: Editorial Universitaria de Buenos Aires, 1960.

KELSEN, H. Justice et Droit naturel. In: AA. VV. Le Droit Naturel. Paris: Presses Universitaires de France, 1959.

LISSKA, A. Aquinas's Theory of Natural Law: An Analytic Reconstruction. Oxford: Clarendon Press, 1996.

MACCORMICK, N. Natural Law Reconsidered. Oxford Journal of Legal Studies, 1, 1981.

MCINERNY, R. Grisez and Thomism. In: Biggar, N. and Black, R. (eds.). The Revival of Natural Law. Ashgat: Aldershot, 2000.

MCINERNY, R. The Principles of Natural Law. The American Journal of Jurisprudence, 25, 1980.

NIELSEN, K. An Examination of the Thomistic Theory of Natural Moral Law. Natural Law Forum. 4, 1959.

NOWELL-SMITH, P. H. Ética. Traducción de Gutiérrez López, G. Estella (Navarra): Editorial Verbo Divino, 1977.

SCHULTZ, J. Is-Ought: Prescribing and a Present Controversy. The Thomist. n. 49, 1985.

VEATCH, H. Natural Law and the 'Is'-'Ought' Question. Catholic Lawyer. 26, 1981.

WIDOW, F. Nominalismo y empirismo en las primeras formulaciones analíticas de la ley de Hume. Convivium. n. 29/30, 2016-2017.

WIDOW, F. La ley de Hume en Hume. La discusión de la interpretación analítica de Treatise III, 1, i. Anales del Seminario de Historia de la Filosofía, Vol. 32. Núm. 2, 2015.

Recebido: 28/01/2019

Aceito: 24/02/2020 
WIDOW, F. 